\title{
Long-term outcome of severe stroke patients : Is the ADL status at discharge from a stroke center indicative of the long-term outcome?
}

\author{
Michiharu Kashihara', Shigetaka Nakao², Jun Kawasaki², Shinjiro Takata², \\ Shinji Nagahiro ${ }^{3}$, Ryuji Kaji ${ }^{4}$, and Natsuo Yasui ${ }^{1}$ \\ ${ }^{1}$ Department of Orthopedics, Institute of Health Biosciences, the University of Tokushima Graduate \\ School, ${ }^{2}$ Division of Rehabilitation, Tokushima University Hospital, ${ }^{3}$ Department of Neurosurgery, \\ and ${ }^{4}$ Department of Neurology, Institute of Health Biosciences, the University of Tokushima Graduate \\ School, Tokushima, Japan
}

\begin{abstract}
The objective of this study was to examine the possibility of predicting the longterm outcome of severe stroke patients from the ADL status at discharge. The design is a retrospective observational study in the stroke center at Tokushima University Hospital. Severe stroke patients at discharge with Barthel Index (BI) scores $\leq 40$ ( $N=51$ ) were divided into two subgroups retrospectively, improved and unimproved. The Functional Independence Measure (FIM) and BI were analyzed at discharge, 3 and 6 months after onset. Correlation analysis between BI and FIM showed good correlation, especially between BI and motor FIM (mFIM). In conclusion, the ADL status at discharge from a stroke center is not definitely indicative of the long-term outcome, although good recovery of severe stroke cases was observed in males and younger patients, and in patients with motor FIM score $>25$. This result shows that the first 3 months after the attack should be regarded as an important time window for intensive rehabilitation. J. Med. Invest. 58 : 227-234, August, 2011
\end{abstract}

Keywords : stroke, outcome, barthel index, functional independence measure, long term

\section{INTRODUCTION}

Recently in Japan, most stroke patients have been transferred to a rehabilitation hospital just after critical acute treatment. The objective of the study was to clarify whether future recovery from a severe condition at discharge is predictable. Nakao et al. (1) used Barthel Index (BI) (2) as an outcome of stroke, which is far simpler than Functional Independence Measure (FIM) (3), and concluded that a

Received for publication May 25, 2011 ; accepted July 20, 2011.

Address correspondence and reprint requests to Michiharu Kashihara, MD, Department of Orthopedics, Institute of Health Biosciences, the University of Tokushima Graduate School, Kuramoto-cho, Tokushima 770-8503, Japan and Fax : +81-88633-7204.
BI score $>40$ at discharge can predict eventual good activities of daily living (ADL). In this study, we used the same database to examine severe stroke patients, BI score $\leq 40$, to explore the possibility of predicting the long-term outcome. This database includes a large number of indicators. For precise examination of the condition, we used motor and cognitive FIM scores combined with BI. The correlation between BI and FIM will also be discussed in detail.

\section{METHODS}

\section{(1) Setting and participants}

In this study, a series of 542 consecutive acute 
stroke inpatients admitted to our hospital in 20072008 was used, and a complete database was gathered for 221 cases (135 infarction, 54 hemorrhage, 29 subarachnoid hemorrhage), from which 51 severe patients (19 infarction, 27 hemorrhage, 5 subarachnoid hemorrhage) with a $\mathrm{BI}$ score $\leq 40$ were selected. Our stroke treatment is based on the Japan Guidelines for the Management of Stroke (4), which is regarded as state of the art. Intravenous tissue plasminogen activator (tPA) can be administered to an ischemic stroke patient within 3 hours after onset. Rehabilitation is initiated within a few days after admission. A seamless relationship between acute and chronic hospitals or clinics was established in 2007 in the Tokushima region. Outcome data were collated and sent on CD-ROM by these treatment facilities. The data were processed according to the ethics rules for the management of personal information.

\section{(2) Outcome measures}

The objective of this study is to study severe and complicated cases with a BI score 0-40 at discharge from the Stroke Center. In this setting, we analyzed $\mathrm{mFIM}$ and cognitive FIM (cFIM) in addition to BI and total FIM (tFIM or FIM) change over time.

\section{(3) Methods of analysis}

The time course, from discharge to 6 months, of the BI score in severe stroke was compared with the mean value. Severe stroke patients were divided into two subgroups : an improved group, which achieved a score $>40$ at 6 months after onset, and an unimproved group. These two groups were compared from the viewpoint of mFIM and cFIM. The contribution of age or gender was additionally analyzed. The correlation between BI and FIM was analyzed using Pearson's correlation coefficient test for total and severe cases separately. Chi-square test was performed to verify independence. All analyses were carried out using Microsoft Excel software (Microsoft Corp., Redmond, WA, USA) add-on software Statcel2 (Oms-publ., Tokorozawa, Japan).

\section{RESULTS}

(1) Correlation analyses between BI and FIM, in total cases and severe cases

We examined Pearson's correlation between BI and $\mathrm{mFIM}$, between BI and tFIM, and between BI and cFIM at discharge in all cases $(\mathrm{N}=542)$. Figure 1
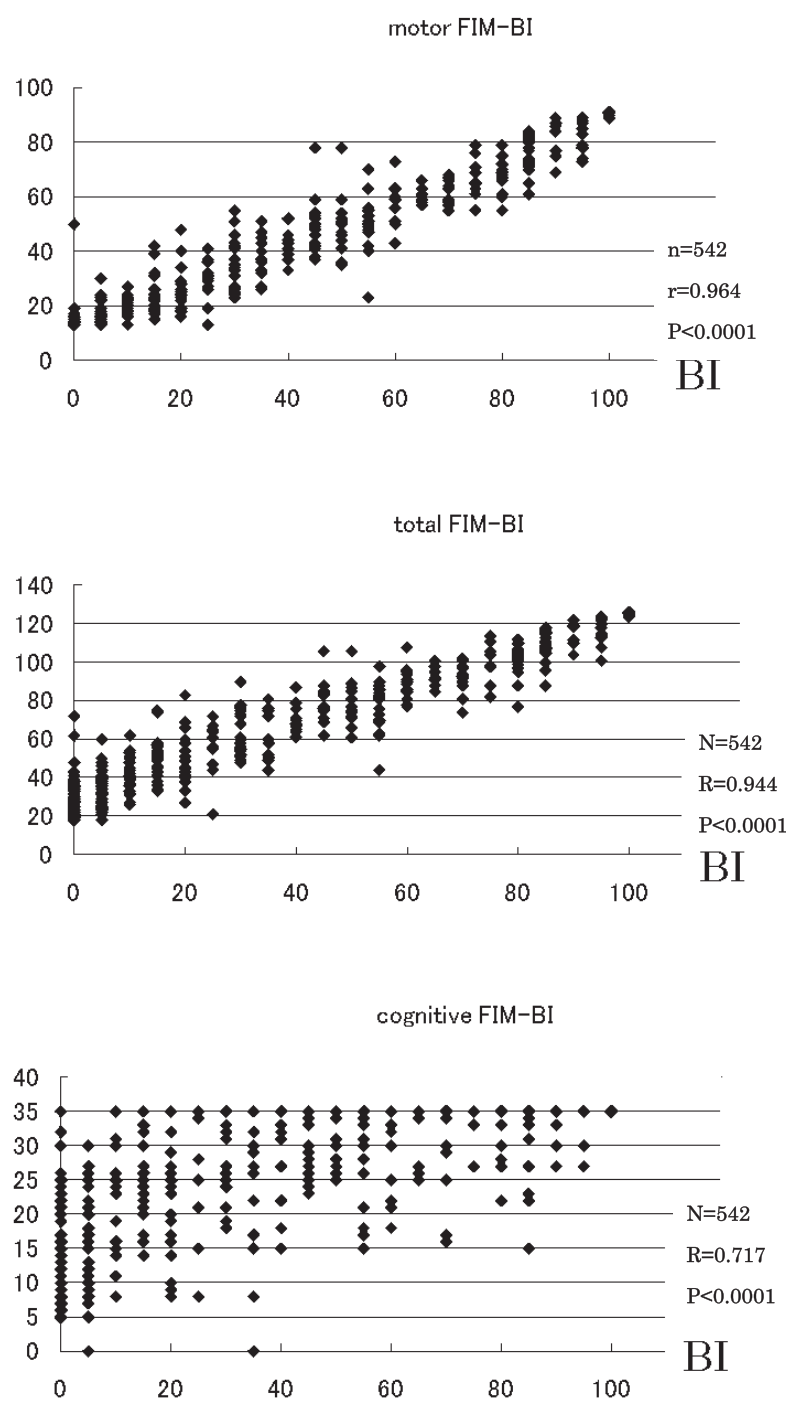

Figure 1. FIM-BI correlation in total cases.

(upper : motor FIM-BI, middle : total FIM-BI, lower : cognitive FIM-BI)

A good correlation between BI and motor FIM ( $r=0.964$, upper) ; $\mathrm{BI}$ and total FIM also correlated with a coefficient $r$ of 0.944 (middle) ; however, BI and cognitive FIM showed a weak correlation $(r=0.717$, lower).

(upper) shows a good correlation between BI and mFIM (coefficient $(r)=0.964)$ and BI and tFIM with a coefficient of 0.944 (Figure 1 (middle)) ; however, $\mathrm{BI}$ and cFIM showed a weaker correlation $(\mathrm{r}=$ 0.717 , Figure 1 (lower)). There was a linear relationship between mFIM and BI, and between tFIM and BI. The following formulas were obtained from single regression analysis :

$\mathrm{mFIM}=0.734 \cdot \mathrm{BI}+13.1(\mathrm{P}$ for $\mathrm{BI}=0 ; \mathrm{P}$ for constant $=1.5 \mathrm{E}-187)$,

tFIM $=0.968 \cdot \mathrm{BI}+27.9(\mathrm{P}$ for $\mathrm{BI}=6 \mathrm{E}-287 ; \mathrm{P}$ for constant $=8.3 \mathrm{E}-187$ ),

where $\mathrm{E}$ is the exponent and $\mathrm{P}$ is the $\mathrm{P}$-value of a t-test. 
We also examined Pearson's correlation in patients with $\mathrm{BI} \leq 40(\mathrm{~N}=51)$. BI and mFIM correlated highly with coefficients of 0.953 on admission, 0.946 at discharge, and 0.974 at 3 months (Table 1). In contrast, mFIM and cFIM correlated with smaller $r$ values of 0.335 on admission, 0.535 at discharge, and 0.721 at 3 months. The severe case group also showed a good correlation between BI and motor FIM, but a weaker correlation between MFIM and cognitive FIM at discharge. The t-test is used to establish the statistical significance of the correlation coefficient. Table 1 shows that the coefficient is highly significant, except between mFIM and cFIM. The correlation between BI (or mFIM) and cognitive FIM was weakened by the group of cases with high cognitive scores and low BI scores in the analysis of total cases, and the correlation was weakened by the group of cases with low cFIM and high BI (or mFIM) scores in severe cases.

\section{(2) Classification of severe cases}

Fifty-one severe cases were divided into two groups : patients who showed improvement after discharge and achieved a BI score of more than 40 (improved group, 28 cases), and those who did not exceed the BI score of 40 until 6 months after onset (unimproved group, 23 cases). The improved group showed gradual recovery of the BI score, and the mean value reached to 60 at 6 months, whereas the unimproved group showed a slight fall at 6 months. These time courses are shown in Figure 2.
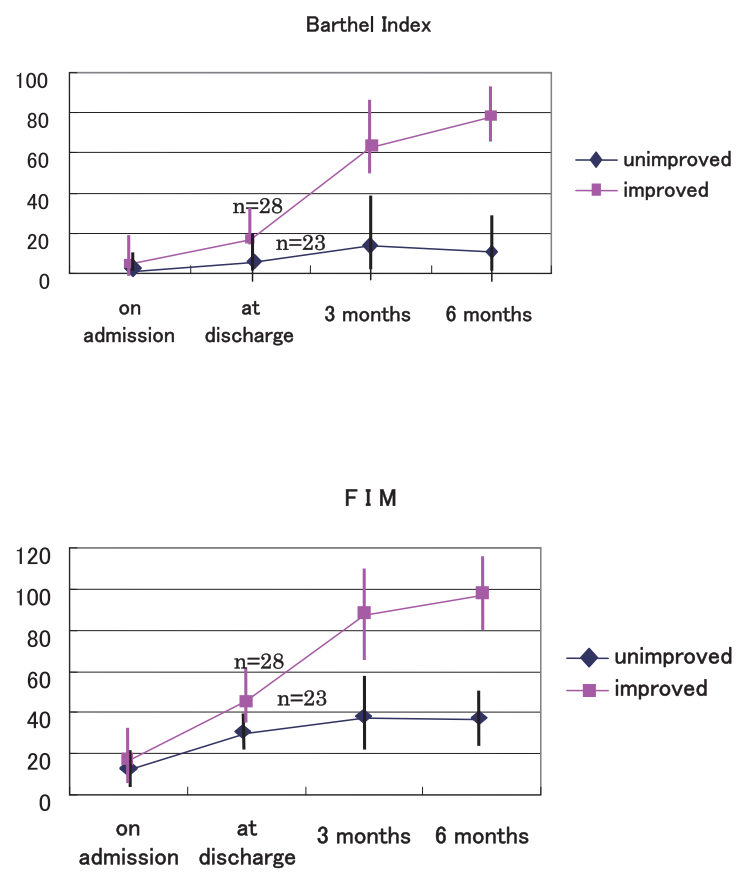

Figure 2. Time course of severe cases in 2 subgroups. (upper : BI, lower : FIM, improved : improved group, unimproved : unimproved group)

The improved group showed gradual recovery of the BI and FIM scores, whereas the unimproved group showed a slight fall at 6 months.

Table 1. Correlation between ADL measures examined in the time course.

(BI : Barthel Index, FIM : Functional Independence Measure, mFIM : motor FIM, tFIM : total FIM, cFIM : cognitive FIM, unimproved : unimproved subgroup, improved : improved subgroup)

$\mathrm{BI}$ and $\mathrm{mFIM}$ exhibit a very close correlation. A smaller correlation is noted between mFIM and cFIM ; the coefficient is especially low in the improved group at 3 months in this table.

\begin{tabular}{|c|c|c|c|c|c|c|}
\hline $\begin{array}{c}\text { day of exam } \\
\text { (subgroup) }\end{array}$ & $\begin{array}{c}\text { Correlation } \\
\text { between }\end{array}$ & cases & $\begin{array}{c}\text { Coefficient } \\
\text { (r) }\end{array}$ & $\mathrm{t}$-value & $\mathrm{P}$-value & $\mathrm{t}(0.975)$ \\
\hline \multirow{3}{*}{$\begin{array}{l}\text { on } \\
\text { admission }\end{array}$} & BI, mFIM & 50 & .953 & 21.8 & $<0.0001$ & 2.00 \\
\hline & BI, tFIM & 50 & .791 & 8.96 & $<0.0001$ & 2.01 \\
\hline & mFIM, cFIM & 50 & .335 & 2.46 & 0.0171 & 2.01 \\
\hline \multirow{3}{*}{$\begin{array}{l}\text { at } \\
\text { discharge }\end{array}$} & BI, mFIM & 51 & .945 & 20.42 & $<0.0001$ & 2.00 \\
\hline & BI, tFIM & 51 & .886 & 13.40 & $<0.0001$ & 2.00 \\
\hline & mFIM, cFIM & 51 & .535 & 4.43 & $<0.0001$ & 2.00 \\
\hline \multirow{3}{*}{$\begin{array}{l}\text { at } \\
3 \text { months }\end{array}$} & BI, mFIM & 45 & .974 & 28.41 & $<0.0001$ & 2.01 \\
\hline & BI, tFIM & 45 & .959 & 22.35 & $<0.0001$ & 2.01 \\
\hline & mFIM, cFIM & 45 & .720 & 6.81 & $<0.0001$ & 2.01 \\
\hline \multirow{3}{*}{$\begin{array}{l}\text { at } \\
3 \text { months } \\
\text { (unimproved) }\end{array}$} & BI, mFIM & 19 & .968 & 16.13 & $<0.0001$ & 2.10 \\
\hline & BI, tFIM & 19 & .929 & 10.42 & $<0.0001$ & 2.10 \\
\hline & mFIM, cFIM & 19 & .724 & 4.33 & 0.0004 & 2.10 \\
\hline \multirow{3}{*}{$\begin{array}{l}\text { at } \\
3 \text { months } \\
\text { (improved) }\end{array}$} & BI, mFIM & 26 & .914 & 11.05 & $<0.0001$ & 2.06 \\
\hline & BI, tFIM & 26 & .878 & 8.99 & $<0.0001$ & 2.06 \\
\hline & mFIM, cFIM & 26 & .388 & 2.06 & 0.0500 & 2.06 \\
\hline
\end{tabular}


(3) Motor FIM and cognitive FIM as indicators of recovery

Motor function and cognitive function at discharge were compared in these two groups, improved and unimproved (Figure 3). In severe stroke cases, cFIM exhibited small scores $\leq 25$ in almost all cases. The patients with $\mathrm{mFIM}>25$ subsequently showed an improving tendency. On the other hand, low scores of mFIM did not always preclude the possibility of recovery in the future. Nearly half of the patients with motor FIM $<25$ showed improvement until 3 to 6 months.

(4) Difference of mFIM-cFIM correlation between improved and unimproved groups

The correlations between mFIM and cFIM at discharge are shown in the lower part of Table 1. In regard to the mFIM-cFIM correlation, the coefficient was 0.724 in the unimproved group, which was higher than that of the improved group (0.388).

\section{(5) Characteristics of severe cases}

(a) Disease distribution

Figure 4 shows the difference in stroke types in severe cases in reference to total cases. In total cases, in the upper panel of the figure, cerebral infarction occupies $60 \%$ of the cases and cerebral hemorrhage is $24 \%$; however, cerebral hemorrhage comprises $53 \%$ of severe cases, in the lower panel
TOTAL CASES $(\mathrm{N}=221)$

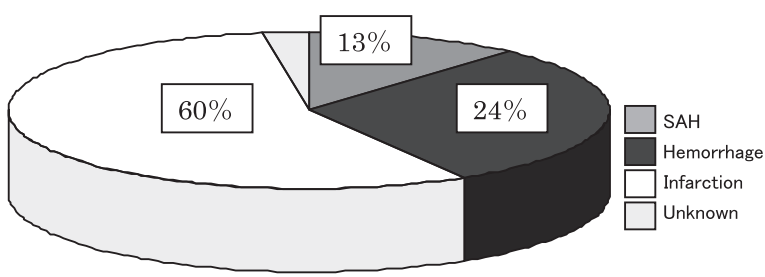

SEVERE CASES: $\mathrm{BI} \leq 40(\mathrm{~N}=51)$

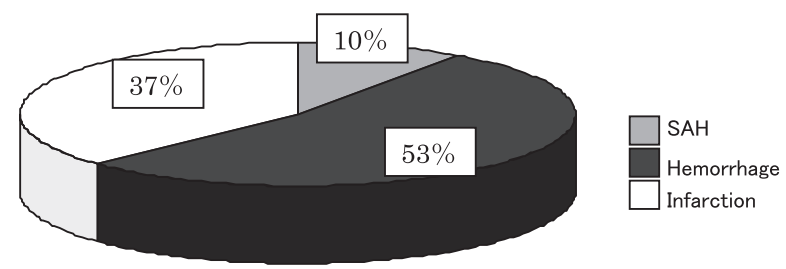

Figure 4. Distribution of the disease. upper : total cases, lower : severe cases Cerebral infarction occupies $60 \%$ in total cases, whereas cerebral hemorrhage increases to $53 \%$ in severe cases.

of the figure. The ratio of infarction/hemorrhage was nearly reversed in severe cases.

(b) Age in the severe group

The average age was $69.8 \pm 23.3$ (SD) in total cases in the stroke center. In severe cases, the average age was $70.5 \pm 10.1$ (SD). There was no difference in age between total cases and severe cases.

\section{cognitive FIM}

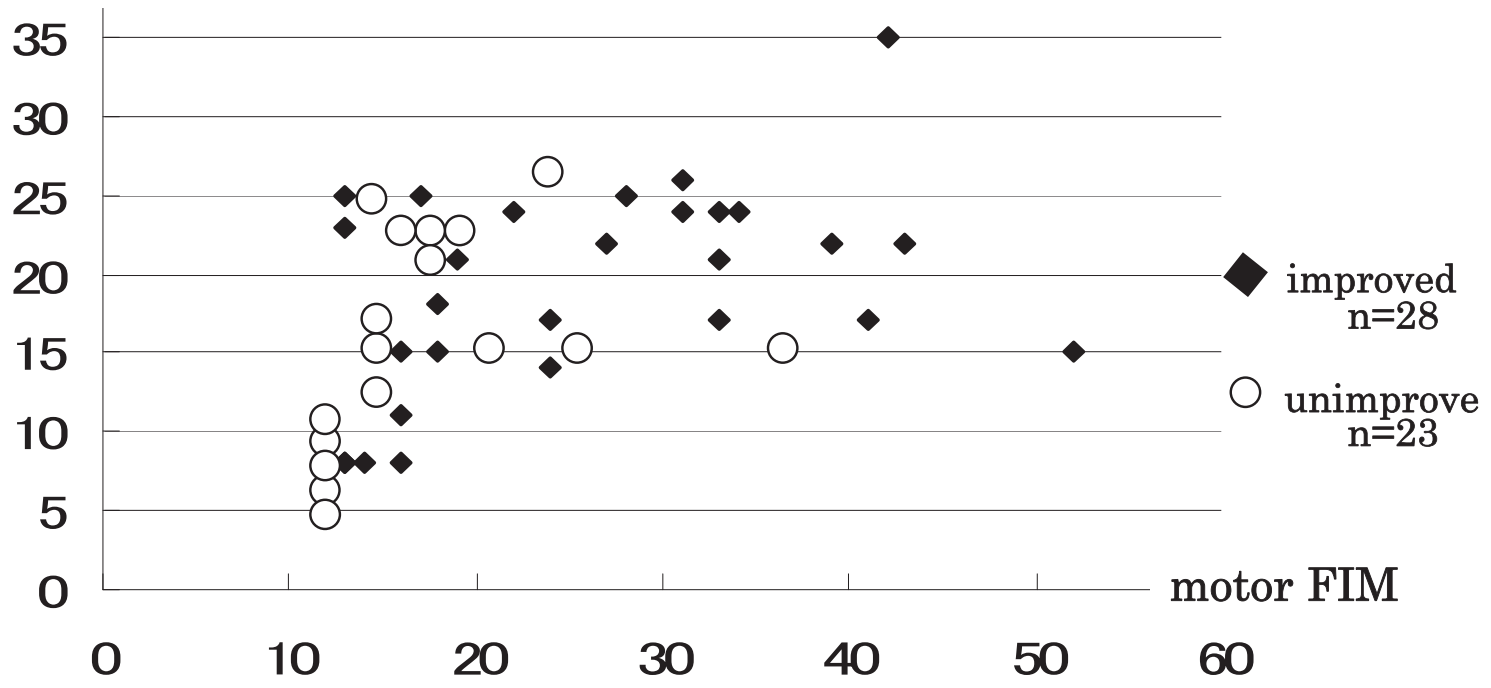

Figure 3. Scatter plot of cognitive FIM-motor FIM in severe stroke cases.

Low scores of motor FIM $\leq 25$ did not preclude the possibility of recovery in the future. Nearly half of the cases showed improvement. 
(6) Comparison between improved group and unimproved group

The improved group was younger $(\mathrm{N}=28$, mean : $66.5 \pm 8.7)$ than the unimproved group $(\mathrm{N}=23$, mean : $76.1 \pm 8.7, \mathrm{P}<0.01$ ).

Figure 5 shows the role of gender in relation to the improvement after severe stroke. There were 23 male patients and 27 female patients. Eighteen male and 10 female patients improved, but 6 male and 17 female patients did not improve. Statistical examination revealed a difference in recovery with regard to gender $\left(\chi^{2}\right.$-test, $\left.\mathrm{P}<0.01\right)$.

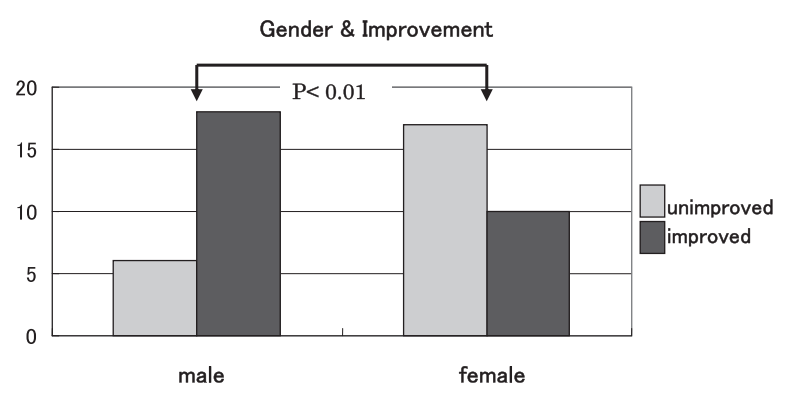

Figure 5. Gender in relation to the improvement after severe stroke.

Males show an improving tendency compared with females.

\section{DISCUSSION}

The BI was devised by Barthel et al. in 1965 (2) and is used mainly in European countries as a simple and useful evaluation of a patient's independence. It can be accurately and quickly scored by adhering to the definition of 10 items concerning $\mathrm{ADL}$, and the score range is $0-100$. FIM was developed by Granger et al. in 1983 for precise evaluation of ADL (3), and is the main tool in the USA (5). FIM is divided into two sections : a motor section, which includes 13 items, and a cognitive section, including 5 items. Each item is graded from 1 (totally dependent) to 7 (completely independent), and the tFIM score range is $18-126$ (mFIM : 13-91, cFIM : 5-35). Many studies have compared the BI and FIM (6-10). Although recent studies from North America were more likely to use the FIM (11), the BI is a simple method that has been used more often than FIM. Our study showed a close correlation between BI and FIM scores; in particular, BI scores and mFIM scores were very closely correlated (Table 1$)$. From this standpoint, BI holds a dominant place because of its simplicity, whereas the FIM is useful to assess cognitive function separately.

The Deming cycle, plan-do-study-action, is a wellknown system for total quality control. In the rehabilitation process of stroke, it is also important to plan suitable action according to the patient's ADL. A large proportion of the literature is concerned with the prediction of post-stroke disability $(1,11$ 17). Recent specific therapy and interventions are said to be associated with better outcomes. Our Stroke Center was established in 2005 with stateof-the-art treatment, including rehabilitation. It may be reasonable to discuss the possibility of prediction in these settings. We analyzed the database of Stroke Center inpatients during 2007-2008, with follow-up until 6 months after onset. Nakao et al. (1), who used the same database, found that a BI score $>40$ at discharge is an excellent indicator of better prognosis in the chronic stage (Figure 6). Then, we focused on severe stroke patients with a BI score $\leq 40$. These patients were a mixture of with and without good recovery. The main objective of the study was to clarify whether further classification of severe cases in regard to the outcome is possible.

The correlation between BI and cFIM was weakened by two groups of cases, one with high cognitive scores and low BI scores, and the other with low cFIM and high BI scores. We consider that these two groups seem to correspond to lacunar infarction and dementia, respectively, because cognitive function is preserved in most lacunar infarctions

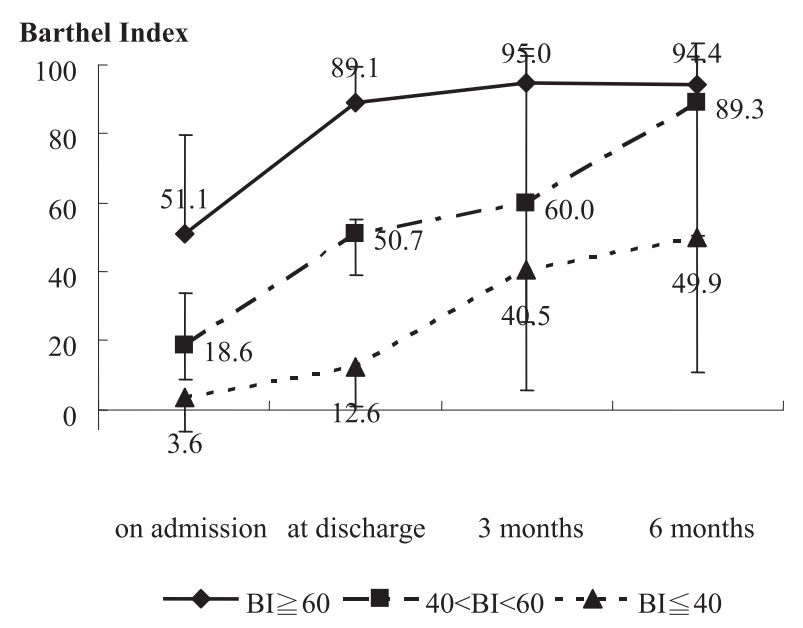

Figure 6. Changes of Barthel Index (BI) in each score range (Nakao et al. 2010, with permission from The Journal of Medical Investigation)

Although all ranges improved, patients with a $\mathrm{BI}>40$ on discharge improved more than patients with a $\mathrm{BI}<40$. Patients with a $\mathrm{BI}>60$ on discharge maintained the same level at 6 months. 
(18) and individuals with dementia sometimes suffer strokes ; therefore, lacunar infarction and dementia should be taken into consideration in BI-cFIM interaction.

In severe cases, cFIM scores were mostly $\leq 25$, both in the improved group and in the unimproved group. Higher mFIM scores, $>25$, showed a recovery tendency ; however, low $\mathrm{mFIM}$ scores, $<25$, did not always indicate no change. Almost half of the patients with $\mathrm{mFIM}$ scores $<25$ belonged to the improved group (Figure 3 ). In mild stroke, good cognitive function seems to facilitate better recovery (1) ; however, our study of severe stroke showed that cFIM did not correlate with better prognosis. In severe stroke, cognitive failure is a common feature and the recovery seems to be slow or difficult $(19,20)$. Speech therapy and rehabilitation for higher brain dysfunction, which are now used in our facilities, have a limit to achieve useful recovery of ADL. More specialized long-term rehabilitation should be applied to regain cognitive function.

It is fundamentally important to reduce cases of severe motor deficit in acute-stage treatment, or more fundamentally to prevent stroke. Until such an innovation, the most important procedure is aggressive rehabilitation for at least 3 months, and if possible, more than 6 months after the onset of stroke (21). The capability of severe stroke patients is not completely predicted from the $\mathrm{ADL}$ at the stage of discharge from a stroke center. Treatment at a stroke center produces immediate recovery in some cases, but in others the symptoms subside gradually after discharge. Good recovery is initiated as a result of early rehabilitation in the acute stage, and strengthened by following rehabilitation for 3 to 6 months. We regard the first 3 months after onset as an important period for intensive rehabilitation, and the rehabilitation team should work hard during this period, regardless of the ADL level at discharge, until a more effective method is developed in the future $(19,22,23)$.

Statistical examination showed that male and younger patients have a greater possibility of recovery. The reason why gender and age play roles in the process of recovery from severe stroke is unknown, but muscular power and balance activity seem to play important roles in the rehabilitation and recovery process. Roth et al. evaluated gender differences in 1-year outcomes for stroke survivors and concluded women are at heightened risk for poor outcomes (24). Kay et al. investigated nontraumatic spinal cord injury and found men were more independent in mobility than women in the most disabled group (25).

\section{LIMITATIONS}

The number of severe cases is insufficient for study subgroups, for example, the type of disease, location of the lesion, and age groups. This suggests that larger studies with tighter controls on the case mix may provide additional information on stroke patients. This study will assist in the interpretation of BI and FIM scores and the direction of stroke therapy in the near future.

This study is a statistical study to predict outcome. Case studies are valuable to estimate the individual outcome utilizing MRI tractography, evoked potentials, and actigraphy, which show the possibility of recovery after the reduction of brain edema after the attack (15-16, 26-29).

\section{CONCLUSIONS}

In severe stroke patients, no accurately predictive ADL factor for good recovery was found at discharge from the stroke center. Although patients with mFIM scores $>25$, male and young showed a recovery tendency, low mFIM scores $<25$ do not always preclude improvement. Almost half of the patients with $\mathrm{mFIM}$ scores $\leq 25$ belonged to the improved group. There is hope for good recovery in a severe stroke patient during the first 3 to 6 months after the attack. We should continue to do our best for patient rehabilitation during this period.

\section{ACKNOWLEDGEMENTS}

The authors are grateful to the staff of the stroke care units and rehabilitation at Tokushima University Hospital, and the many therapists who persisted energetically with rehabilitation after patient discharge from our hospital. The authors are deeply indebted to Masayasu Matsumoto from Hiroshima University for academic writing.

\section{REFERENCES}

1. Nakao S, Takata S, Kashihara M, Nagahiro S, Kaji R, Yasui N : Relationship between Barthel 
Index scores during the acute phase of rehabilitation and subsequent ADL in stroke patients. J Med Invest 57 : 81-88, 2009

2. Mahoney FI, Barthel DW : Functional Evaluation : Barthel Index. Md State Med J 14 : 6165, 1965

3. Granger CV, Hamilton BB, Gresham GE : The stroke rehabilitation outcome study-Part I : General description. Arch Phys Med Rehabil 69 : 506-509, 1988

4. The Joint Committee on guidelines for the Management of Stroke, In : Japanese Guidelines for the Management of Stroke, Kyowa Kikau, Ltd, Tokyo, 2004, pp.173-174

5. Sangha H, Lipson D, Foley N, Salter K, Bhogal S, Pohani G, Teasell RW : A comparison of the Barthel Index and the Functional Independence Measure as outcome measures in stroke rehabilitation : patterns of disability scale usage in clinical trials. Int J Rehabil Res. 28 : 135-139, 2005

6. Kwon S, Hartzema AG, Duncan PW, Min-Lai $\mathrm{S}$ : Disability measures in stroke. Relationship among the Barthel Index, the Functional Independence Measure, and the Modified Rankin Scale. Stroke 35 : 918-923, 2004

7. Kasner SE : Clinical interpretation and use of stroke scales. Lancet Neurol 5 : 603-612, 2006

8. Wallace D, Duncan PW, Lai SM : Comparison of the responsiveness of the Barthel Index and the motor component of the Functional Independence Measure in stroke : the impact of using different methods for measuring responsiveness. J Clin Epidemiol 55 : 922-928, 2002

9. Dromerick AW, Edwards DF, Diringer MN : Sensitivity to changes in disability after stroke: a comparison of four scales useful in clinical trials. J Rehabil Res Dev 40 : 1-8, 2003

10. Kidd D, Steward G, Baldry J, Johnson J, Rossiter D, Petruckevitch A, Thompson AJ : The functional independence measure : a comparative validity and reliability study. Disabil Rehabil $17:$ 10-14, 1995

11. Kawakkel G, Wagenaar RC, Kollen BJ, Lankhorst GJ : Predicting disability in strokeA critical review of the literature. Age and Ageing 25 : 479-489, 1996

12. Aprile I, Di Stasio E, Romitelli F, Lancellotti S, Caliandro P, Tonali P, Gilardi A, Padua L: Effects of rehabilitation on quality of life in patients with chronic stroke. Brain Inj $22: 451-6$, 2008
13. Protopsaltis J, Kokkoris S, Korantzopoulos P, Milionis HJ, Karzi E, Anastasopoulou A, Filioti $\mathrm{K}$, Antonopoulos S, Melidonis A, Giannoulis $\mathrm{G}$ : Prediction of long-term functional outcome in patients with acute ischemic non-embolic stroke. Atherosclerosis $203: 228-35,2009$

14. Pan SL, Wu SC, Lee TK, Chen TH : Reduction of disability after stroke is a more informative predictor of long-time survival than initial disability status. Disabil Rehabil 29:417-423, 2007

15. Sonoda S, Saitoh E, Nagai S, Okuyama Y, Suzuki T, Suzuki M. Stroke outcome prediction using reciprocal number of initial activities of daily libing status. J Stroke Cerebrovasc Dis $14: 8-11,2005$

16. Al-Rawi MA, Hamdan FB, Abdul-Muttalib AK : Somatosensory evoked potentials as a predictor for functional recovery of the upper limb in patients with stroke. J Stroke Cerebrovasc Dis $18: 262-268,2009$

17. Pizzi A, Carrai R, Falsini C, Martini M, Verdesca S, Grippo A : Prognostic value of motor evoked potentials in motor function recovery of upper limb after stroke. J Rehabil Med 41: 654-660, 2009

18. Kashihara M, Matsumoto K : Acute capsular infarction. Location of the lesion and the clinical features. Neuroradiology $27: 248$-253,1985

19. Rigby H, Gubitz G, Reidy Y, Christian C, Grover V Phillips S : Caring for stroke survivors : baseline and 1-year determinants of caregiver burden. Int J Stroke 4 : 152-158, 2009

20. Wilz G : Predictors of subjective impairment after stroke : influence of depression, gender and severity of stroke. Brain Inj 21 : 39-45, 2007

21. Horn SD, DeJong G, mout RJ, Gassaway J, James R, Conroy B : Stroke rehabilitation patients, practice, and outcomes : is earlier and more aggressive therapy better? Arch Phys Med Rehabil 86(12 Suppl 2) : S101-114, 2005

22. Rigby H, Gubitz G, Reidy Y, Christian C, Grover V Phillips S : Caring for stroke survivors : baseline and 1-year determinats of caregiver burden. Int J Stroke $4: 152-8,2009$

23. Hinkle JL, Davies J, Ng D, McClaran J : Examining assessment tools for discharge planning. Nurs Times $104:$ 32-35, 2008

24. Roth DL, Haley WE, Cley OJ, Perkins M, Grant JS, Rhodes JD, Wadley VG, Kissela B, Howard $\mathrm{G}$ : Race and gender differences in 1-year outcomes for community-dwelling stroke servivors 
with family caregivers. Stroke $42: 626-631$, 2011

25. Kay E, Deutsch A, Chen D, Semik P, Rowles $\mathrm{D}$ : Effects of gender on inpatients rehabilitation outcomes in the elderly with incomplete paraplegia from montraumatic spinal cord injury. $\mathrm{J}$ Spinal Cord Med 33 : 379-386, 2010

26. Walker MF, Leonardi-Bee J, Langhorne $P$, Dewey M, Drummond A, Gilbertson L, Gladman JRF, Jongbloed L, Logan P, Parker $\mathrm{C}$ : Individual patient data meta-analysis of randomized controlled trials of community occupational therapy for stroke patients. Stroke 35 : 2226-2232, 2004
27. Konishi J, Yamada K, Kizu O, Ito H, Sugimura K, Yoshikawa K, Nakagawa M, Nishimura T: MR tractography for the evaluation of functional recovery from lenticulostriate infarcts. Neurology $11: 108-13,2005$

28. Newton JM, Ward NS, Parker GJ, Deichmann R, Alexander DC, Friston KJ, Frackowiak RS : Non-invasive mapping of corticofugal fibres from multiple motor areas-relevance to stroke recovery. Brain $129:$ 1844-1858, 2006

29. Reiterer V, Sauter C, Kloesch G, Lalouschek W, Zeitlhofer J : Actigraphy-a useful tool for motor activity monitoring in stroke patients. Eur Neurol 60 : 285-291, 2008 